\title{
Some results on a certain type of difference equation originated from difference Painlevé I equation
}

Qian $\mathrm{Li}^{1}$ and Zhi-Bo Huang ${ }^{2^{*}}$

\section{"Correspondence:}

huangzhibo@scnu.edu.cn

${ }^{2}$ School of Mathematical Sciences,

South China Normal University,

Guangzhou, 510631, P.R. China

Full list of author information is

available at the end of the article

\section{照 Springer}

\begin{abstract}
In this paper, by using Nevanlinna value distribution theory, we consider a certain type of difference equation, which originates with the difference Painlevé I equation, $f(z+1)+f(z-1)=\frac{A(z)}{f(z)}+C(z)$, where $A(z), C(z)$ are small meromorphic functions relative to $f(z)$, and we obtain the existence and the forms of rational solutions. We also discuss the properties of the Borel exceptional value, zeros, poles, and fixed points of finite order transcendental meromorphic solutions.
\end{abstract}

MSC: 30D35; 39B32; 34M05

Keywords: difference equation; meromorphic solution; rational solution

\section{Introduction}

In this paper, a meromorphic function means meromorphic in the whole complex plane $\mathbb{C}$. We assume that the reader is familiar with the standard symbols and fundamental results of Nevanlinna theory; see e.g. [1, 2]. For a meromorphic function $f(z)$, let $\sigma(f)$ be the order of growth of $f(z)$. Further, let $\lambda(f)$ (resp. $\lambda(1 / f)$ ) be the exponent of convergence of the zeros (resp. poles) of $f(z)$. We also use the notation $\tau(f)$ to denote the exponent of convergence of fixed points of $f(z)$, which is defined by

$$
\tau(f)=\varlimsup_{r \rightarrow \infty} \frac{\log N\left(r, \frac{1}{f(z)-z}\right)}{\log r} .
$$

Moreover, we use the notation $\operatorname{deg}_{f} R(z, f)$ for the degree of a rational function $R(z, f)$ with respect to $f(z)$, where $R(z, f)$ is rational in both of its arguments with small functions relative to $f(z)$ as its coefficients. In what follows $\mathfrak{F}(f)$ denotes the field of small functions relative to $f(z)$.

Halburd and Korhonen [3] used ideas related to the singularity confinement test [4] in its proof, and considered a difference equation of type

$$
f(z+1)+f(z-1)=R(z, f) .
$$

The solutions of (1.1) are called admissible. If (1.1) has at least one admissible meromorphic solution of finite order, they both showed that either $f(z)$ satisfies a difference Riccati

(c) $2015 \mathrm{Li}$ and Huang. This article is distributed under the terms of the Creative Commons Attribution 4.0 International License (http://creativecommons.org/licenses/by/4.0/), which permits unrestricted use, distribution, and reproduction in any medium, provided you give appropriate credit to the original author(s) and the source, provide a link to the Creative Commons license, and indicate if changes were made. 
equation, or (1.1) can be transformed into a difference Painlevé or a linear equation. Now, we recall their result.

Theorem 1.A (Theorem 1.1 of [3]) If the equation

$$
f(z+1)+f(z-1)=R(z, f),
$$

where $R(z, f)$ is rational in $f(z)$ and meromorphic in $z$, has an admissible meromorphic solution of finite order, then either $f(z)$ satisfies a difference Riccati equation,

$$
f(z+1)=\frac{\bar{p} f(z)+q}{f(z)+p}
$$

where $p, q \in \mathfrak{F}(f)$, or (1.1) can be transformed by a linear change in $f(z)$ into one of the following equations:

$$
\begin{aligned}
& f(z+1)+f(z)+f(z-1)=\frac{\pi_{1} z+\pi_{2}}{f(z)}+\kappa_{1}, \\
& f(z+1)-f(z)+f(z-1)=\frac{\pi_{1} z+\pi_{2}}{f(z)}+(-1)^{z} \kappa_{1}, \\
& f(z+1)+f(z-1)=\frac{\pi_{1} z+\pi_{3}}{f(z)}+\pi_{2} \\
& f(z+1)+f(z-1)=\frac{\pi_{1} z+\kappa_{1}}{f(z)}+\frac{\pi_{2}}{f(z)^{2}} \\
& f(z+1)+f(z-1)=\frac{\left(\pi_{1} z+\kappa_{1}\right) f(z)+\pi_{2}}{(-1)^{-z}-f(z)^{2}} \\
& f(z+1)+f(z-1)=\frac{\left(\pi_{1} z+\kappa_{1}\right) f(z)+\pi_{2}}{1-f(z)^{2}} \\
& f(z+1) f(z)+f(z) f(z-1)=p \\
& f(z+1)+f(z-1)=p f(z)+q
\end{aligned}
$$

where $\pi_{k}, \kappa_{k} \in \mathfrak{F}(f)$ are arbitrary finite order periodic functions with period $k$.

Recently, due to the difference analog of the lemma on the logarithmic derivative given by Halburd and Korhonen in [5], and Chiang and Feng in [6] independently, many authors focused their interest on the complex difference analogs of Nevanlinna theory and complex difference equations (see [5-13]). But most of them mainly dealt with the growth of order of meromorphic solutions of difference equations (see e.g. $[6,9,13]$ ).

Though there are few papers on the existence of finite order meromorphic solution of difference equations (see $[3,9,11,12])$, there is only one paper concerning with the existence of rational solution of difference Painlevé I equation (see [9]). In this paper, we will discuss the existence and forms of rational solutions, and investigate the properties on finite order transcendental meromorphic solutions of a certain type of difference equation originating with the difference Painlevé I equation. 


\section{The existence and forms of rational solutions}

Chen and Shon considered the difference Painlevé I equation

$$
f(z+1)+f(z-1)=\frac{a z+b}{f(z)}+c
$$

and obtained the following result.

Theorem 2.A (Theorem 4 of [9]) Let $a, b, c$ be constants, where $a, b$ are not both equal to zero. Then

(i) if $a \neq 0$, then (2.1) has no rational solution;

(ii) if $a=0$, and $b \neq 0$, then (2.1) has a nonzero constant solution $f(z)=A$, where $A$ satisfies

$$
2 A^{2}-c A-b=0
$$

The other rational solution $f(z)$ satisfies $f(z)=\frac{P(z)}{Q(z)}+A$, where $P(z)$ and $Q(z)$ are relatively prime polynomials and satisfy $\operatorname{deg} P<\operatorname{deg} Q$.

What will happen if we consider a certain type of difference equation originating with the difference Painlevé I equation (2.1)? Here, we obtain the following result.

Theorem 2.1 Let C be a nonzero constant, and $A(z)=\frac{m(z)}{n(z)}$ be an irreducible rational function, where $m(z)$ and $n(z)$ are polynomials with $\operatorname{deg} m(z)=m$ and $\operatorname{deg} n(z)=n$.

(i) Suppose that $m \geq n$ and $m-n$ is an even number or zero. If the difference equation

$$
f(z+1)+f(z-1)=\frac{A(z)}{f(z)}+C
$$

has an irreducible rational solution $f(z)=\frac{P(z)}{Q(z)}$, where $P(z)$ and $Q(z)$ are polynomials with $\operatorname{deg} P(z)=p$ and $\operatorname{deg} Q(z)=q$, then

$$
p-q=\frac{m-n}{2}
$$

(ii) Suppose that $m<n$. If the difference equation (2.2) has an irreducible rational solution $f(z)=\frac{P(z)}{Q(z)}$, then

$$
q-p=n-m \geq 1 \quad \text { or } \quad q-p=0 .
$$

(iii) Suppose that $m>n$ and $m-n$ is an odd number.

Then the difference equation (2.2) has no rational solution.

Remark 2.1 We know that (1.2), (1.3), and (1.4) are difference Painlevé I equations. Why do we only consider the existence and forms of rational solutions of (1.3)? We cannot know the limits of the type $0 \cdot \infty$ when we investigate (1.2) and (1.4) by using the same method below.

Example 2.1, Example 2.2, and Example 2.3 show that the difference equations have rational solutions satisfying Theorem 2.1(i), and Example 2.4 and Example 2.5 show that the difference equations have rational solutions satisfying Theorem 2.1(ii). 
Example 2.1 The difference equation

$$
f(z+1)+f(z-1)=\frac{2 z^{2}-C z}{f(z)}+C
$$

has a rational solution $f(z)=z$, where $m=2, n=0, p=1, q=0$, and $p-q=1=\frac{m-n}{2}$.

Example 2.2 The difference equation

$$
f(z+1)+f(z-1)=\frac{\frac{2 z^{5}-C z^{4}+2 z^{3}+C}{z^{3}-z}}{f(z)}+C
$$

has a rational solution $f(z)=z+\frac{1}{z}$, where $m=5, n=3, p=2, q=1$, and $p-q=1=\frac{m-n}{2}$.

Example 2.3 The difference equation

$$
f(z+1)+f(z-1)=\frac{\frac{(2-C) z^{3}+(2+C) z^{2}-2(2-C) z-4}{z^{3}-3 z^{2}+2 z}}{f(z)}+C
$$

has a rational solution $f(z)=\frac{z+1}{z-1}$, where $C \neq 2, m=n=3, p=q=1$, and $p-q=0=\frac{m-n}{2}$.

Example 2.4 The difference equation

$$
f(z+1)+f(z-1)=\frac{\frac{-C z^{2}+2 z+C}{z^{3}-z}}{f(z)}+C
$$

has a rational solution $f(z)=\frac{1}{z}$, where $C \neq 0, m=2, n=3, p=0, q=1$, and $q-p=1=n-m$.

Example 2.5 The difference equation

$$
f(z+1)+f(z-1)=\frac{\frac{4(z+1)}{z(z-2)}}{f(z)}+2
$$

has a rational solution $f(z)=\frac{z+1}{z-1}$, where $m=1, n=2, p=q=1$, and $q-p=0$.

Proof of Theorem 2.1 Suppose that $f(z)=\frac{P(z)}{Q(z)}$ is a rational solution of (2.2). Then $f(z)$ can be written as

$$
\begin{aligned}
f(z)= & \frac{P(z)}{Q(z)} \\
= & \sum_{j=1}^{k}\left[\frac{c_{j \lambda_{j}}}{\left(z-z_{j}\right)^{\lambda_{j}}}+\frac{c_{j \lambda_{j-1}}}{\left(z-z_{j}\right)^{\lambda_{j-1}}}+\cdots+\frac{c_{j 1}}{z-z_{j}}\right] \\
& +B_{0}+B_{1} z+\cdots+B_{\nu} z^{\nu},
\end{aligned}
$$

where $c_{j \lambda_{j}}(\neq 0), c_{j \lambda_{j-1}}, \ldots, c_{j 1}(j=1,2, \ldots, k)$, and $B_{0}, B_{1}, \ldots, B_{v}$ are constants, $z_{j}(j=1,2, \ldots, k)$ are poles of $f(z)$ with multiplicities $\lambda_{j}$, respectively. 
(i) Suppose that $m>n$ and $m-n$ is an even number. We conclude from (2.2) and (2.3) that

$$
\frac{P(z)}{Q(z)}\left[\frac{P(z+1)}{Q(z+1)}+\frac{P(z-1)}{Q(z-1)}\right]=\frac{m(z)}{n(z)}+C \frac{P(z)}{Q(z)}
$$

If $\operatorname{deg} P(z)=p<q=\operatorname{deg} Q(z)$, then $\frac{P(z)}{Q(z)} \rightarrow 0, \frac{P(z+1)}{Q(z+1)} \rightarrow 0$, and $\frac{P(z-1)}{Q(z-1)} \rightarrow 0$ as $z \rightarrow \infty$, while $\frac{m(z)}{n(z)} \rightarrow \infty$ as $z \rightarrow \infty$. Thus, (2.4) is a contradiction.

If $\operatorname{deg} P(z)=p=q=\operatorname{deg} Q(z)$, then $\frac{P(z)}{Q(z)} \rightarrow a, \frac{P(z+1)}{Q(z+1)} \rightarrow a$, and $\frac{P(z-1)}{Q(z-1)} \rightarrow a$ as $z \rightarrow \infty$, where $a$ is a nonzero constant, while $\frac{m(z)}{n(z)} \rightarrow \infty$ as $z \rightarrow \infty$. Thus, (2.4) is also a contradiction.

If $\operatorname{deg} P(z)=p>q=\operatorname{deg} Q(z)$, then we can assume that $B_{\mu} \neq 0$ and $B_{j} \equiv 0(1 \leq \mu \leq v$, $\mu<j \leq v)$. We obtain, by (2.3), for all sufficiently large $z$,

$$
\left\{\begin{array}{l}
f(z)=B_{\mu} z^{\mu}\left(1+o\left(z^{-1}\right)\right) \\
f(z+1)=B_{\mu} z^{\mu}\left(1+o\left(z^{-1}\right)\right) \\
f(z-1)=B_{\mu} z^{\mu}\left(1+o\left(z^{-1}\right)\right) \\
A(z)=\frac{m(z)}{n(z)}=A z^{m-n}\left(1+o\left(z^{-1}\right)\right)
\end{array}\right.
$$

where $A$ is a nonzero constant. Therefore, we obtain from (2.2), for all sufficiently large $z$,

$$
2 B_{\mu}^{2} z^{2 \mu}\left(1+o\left(z^{-1}\right)\right)=A z^{m-n}\left(1+o\left(z^{-1}\right)\right)+C B_{\mu} z^{\mu}\left(1+o\left(z^{-1}\right)\right)
$$

Thus, we deduce from (2.5) that

$$
p-q=\mu=\frac{m-n}{2}
$$

Now we suppose that $m=n$. So, for all sufficiently large $z$,

$$
A(z)=\frac{m(z)}{n(z)}=A^{*}\left(1+o\left(z^{-1}\right)\right)
$$

where $A^{*}$ is a nonzero constant.

If $\operatorname{deg} P(z)=p<q=\operatorname{deg} Q(z)$, then using the same method as above, we get a contradiction.

If $\operatorname{deg} P(z)=p>q=\operatorname{deg} Q(z)$, using the same method as above, we deduce that, for all sufficiently large $z$,

$$
2 B_{\mu}^{2} z^{2 \mu}\left(1+o\left(z^{-1}\right)\right)=A^{*}+C B_{\mu} z^{\mu}\left(1+o\left(z^{-1}\right)\right)
$$

This is a contradiction. Hence,

$$
p-q=0=\frac{m-n}{2}
$$

From the above, if $m \geq n$ and $m-n$ is an even number or zero, $f(z)=\frac{P(z)}{Q(z)}$ is a rational solution of (2.2), the degree of $P(z)$ and $Q(z)$ satisfy

$$
p-q=\frac{m-n}{2}
$$


(ii) Suppose that $m<n$. Then, for all sufficiently large $z$, we get

$$
A(z)=\frac{m(z)}{n(z)} \rightarrow 0
$$

If $\operatorname{deg} P(z)=p>q=\operatorname{deg} Q(z)$, then we can assume that $B_{\mu} \neq 0$ and $B_{j} \equiv 0(1 \leq \mu \leq v$, $\mu<j \leq v)$. Using the same method as above, we deduce that, for all sufficiently large $z$,

$$
2 B_{\mu}^{2} z^{2 \mu}\left(1+o\left(z^{-1}\right)\right)=C B_{\mu} z^{\mu}\left(1+o\left(z^{-1}\right)\right) .
$$

This is a contradiction.

If $\operatorname{deg} P(z)=p<q=\operatorname{deg} Q(z)$, we conclude from $f(z)=\frac{P(z)}{Q(z)}$ and (2.2) that

$$
\begin{gathered}
m(z) Q(z) Q(z+1) Q(z-1)+C n(z) P(z) Q(z+1) Q(z-1) \\
-n(z) P(z)[P(z+1) Q(z-1)+P(z-1) Q(z+1)]=0 .
\end{gathered}
$$

So, (2.7) yields

$$
\left\{\begin{array}{l}
\operatorname{deg} n(z) P(z)[P(z+1) Q(z-1)+P(z-1) Q(z+1)]=n+2 p+q \\
\operatorname{deg} m(z) Q(z) Q(z+1) Q(z-1)=m+3 q \\
\operatorname{deg} C n(z) P(z) Q(z+1) Q(z-1)=n+p+2 q
\end{array}\right.
$$

Now, we compare the degree of three terms in (2.7). If $m+2 p+q=m+3 q$, then $q-p=$ $\frac{n-m}{2}$. When $n-m$ is an odd number, it is a contradiction obviously. When $n-m$ is an even number, we conclude that

$$
(n+p+2 q)-(m+3 q)=\frac{n-m}{2}>0 \quad \text { and } \quad(n+p+2 q)-(n+2 p+q)=\frac{n-m}{2}>0 .
$$

This shows that there is only one term $-\operatorname{Cn}(z) P(z) Q(z+1) Q(z-1)$ in (2.7) which has the highest degree. This is also a contradiction. If $m+2 p+q=n+p+2 q$, then $q-p=0$. This is a contradiction since $p<q$. If $m+3 q=n+p+2 q$, then $q-p=n-m \geq 1$ and $(m+3 q)-(n+2 p+q)=q-p \geq 1$.

If $\operatorname{deg} P(z)=p=q=\operatorname{deg} Q(z)$, then we may assume that $B_{0} \neq 0$ and $B_{j}=0(j=1,2, \ldots, v)$.

Thus, for all sufficiently large $z$, we have

$$
\left\{\begin{array}{l}
f(z)=B_{0}\left(1+o\left(z^{-1}\right)\right) \\
f(z+1)=B_{0}\left(1+o\left(z^{-1}\right)\right) \\
f(z-1)=B_{0}\left(1+o\left(z^{-1}\right)\right)
\end{array}\right.
$$

Therefore we obtain from (2.2) and (2.6), for all sufficiently large $z$,

$$
2 B_{0}^{2}\left(1+o\left(z^{-1}\right)\right)=C B_{0}\left(1+o\left(z^{-1}\right)\right) .
$$

This means that $p=q$ is possible if

$$
\lim _{z \rightarrow \infty} \frac{P(z)}{Q(z)}=\frac{C}{2} .
$$


Thus, if $n<m$, then the degrees of $P(z)$ and $Q(z)$ satisfy

$$
q-p=n-m \geq 1 \quad \text { or } \quad q-p=0
$$

(iii) Suppose that $n>m$ and $n-m$ is an odd number, and that (2.2) has a rational solution $f(z)=\frac{P(z)}{Q(z)}$. By the proof in (i), we also get $p-q=\frac{m-n}{2}$. This is a contradiction. Thus, (2.2) has no rational solution. The proof of Theorem 2.1 is completed.

\section{Value distribution of finite order meromorphic solutions}

Let $f(z)$ be an admissible meromorphic solution of the equation

$$
f(z+1)+f(z-1)=\frac{A(z)}{f(z)}+C(z)
$$

where $A(z), C(z) \in \mathfrak{F}(f)$. Suppose that there exist $k \geq 1$ and $\alpha<1$ such that

$$
n(f(z+1)+f(z-1)) \leq \alpha n(r+k, f(z))
$$

By the method used in Theorem 2.5 of [3], we see that $f(z)$ is of infinite order of growth.

On the other hand, using similar arguments to those shown in Theorem 3 of [7] and Proposition 8 of [12], if (1.1) admits a finite order non-rational meromorphic solution, then $\operatorname{deg}_{f} R(z, f) \leq 2$. The proofs rely heavily on the Valiron-Mohon'ko lemma, which states that

$$
R(r, f(z))=\operatorname{deg}_{f} T(r, f(z))+O(\log r) .
$$

Now recalling the fact that

$$
T(r, f(z \pm 1)) \leq(1+\varepsilon) T(r+1, f(z))+O(1)
$$

holds for all $\varepsilon>0$, if $r$ is sufficiently large, we conclude that

$$
T(r+1) \geq \frac{\operatorname{deg}_{f(z)} R(z, f(z))}{2(1+\varepsilon)} T(r, f(z))+O(\log r) .
$$

This implies that $f(z)$ is of infinite order unless the degree of $R(z, f)$ is at most two.

The above facts imply that it is possible that (3.1) has finite order transcendental meromorphic solutions. Thus, we consider (3.1) and obtain the following.

Theorem 3.1 Suppose that the equation

$$
f(z+1)+f(z-1)=\frac{A(z)}{f(z)}+C(z)
$$

where $A(z), C(z) \in \mathfrak{F}(f)$, admits a finite order transcendental meromorphic solution $f(z)$. Then:

(i) $\lambda(f)=\lambda\left(\frac{1}{f}\right)=\sigma(f)$.

(ii) $f(z)$ has no Borel exceptional value. 
(iii) If $A(z) \not \equiv 2 z^{2}-z C(z)$, then the exponent of convergence of fixed points of $f(z)$ satisfies $\tau(f)=\sigma(f)$.

We need some lemmas to prove Theorem 3.1.

Lemma 3.1 (Theorem 3.2 of [5]) Let $f(z)$ be a non-constant finite order meromorphic solution of

$$
P(z, f(z))=0,
$$

where $P(z, f(z))$ is a difference polynomial in $f(z)$. If $P(z, a) \not \equiv 0$ for a meromorphic function $a \in \mathfrak{F}(f)$, then

$$
m\left(r, \frac{1}{f(z)-a}\right)=S(r, f) .
$$

Lemma 3.2 (Theorem 2.3 of [13]) Let $f(z)$ be a transcendental meromorphic solution of finite order $\sigma$ of a difference equation of the form

$$
U(z, f) P(z, f)=Q(z, f)
$$

where $U(z, f), P(z, f)$, and $Q(z, f)$ are difference polynomials with all coefficients $\alpha_{\lambda}(z)$ small functions as understood in the usual Nevanlinna theory, i.e. $T\left(r, \alpha_{\lambda}\right)=O\left(r^{\sigma-1+\varepsilon}\right)+S(r, f)$. The maximum total degree is $\operatorname{deg}_{f} U(z, f)=n$ in $f(z)$ and its shifts, and $\operatorname{deg}_{f} Q(z, f) \leq n$. Moreover, we assume that $U(z, f)$ contains just one term of maximal total degree in $f(z)$ and its shifts. Then, for each $\varepsilon>0$,

$$
m(r, P(z, f))=O\left(r^{\sigma-1+\varepsilon}\right)+S(r, f)
$$

possibly outside of an exceptional set of finite logarithmic measure.

Lemma 3.3 (Theorem 2.2 of [6]) Let $f(z)$ be a meromorphic function with exponent of convergence of poles $\lambda\left(\frac{1}{f}\right)=\lambda<+\infty, \eta \neq 0$ be fixed, then, for each $\varepsilon>0$,

$$
N(r, f(z+\eta))=N(r, f)+O\left(r^{\lambda-1+\varepsilon}\right)+O(\log r) .
$$

Lemma 3.4 (Theorem 1.51 of [14]) Suppose that $f_{j}(z)(j=1,2, \ldots, n)(n \geq 2)$ are meromorphic functions, and $g_{j}(z)(j=1,2, \ldots, n)$ are entire functions satisfying the following conditions.

(1) $\sum_{j=1}^{n} f_{j}(z) e^{g_{j}(z)}=0$.

(2) $g_{j}(z)-g_{k}(z)$ are not constants for $1 \leq j<k \leq n$.

(3) For $1 \leq j \leq n, 1 \leq h<k \leq n$,

$$
T\left(r, f_{j}\right)=o\left\{T\left(r, e^{g_{h}-g_{k}}\right)\right\} \quad(r \rightarrow+\infty, r \notin E),
$$

where $E \subset(1,+\infty)$ is of finite linear measure or finite logarithmic measure.

Then $f_{j}(z) \equiv 0(j=1,2, \ldots, n)$. 
Proof of Theorem 3.1 (i) Suppose that $f(z)$ is a finite order transcendental meromorphic solution of (3.1). We obtain from (3.1)

$$
P_{1}(z, f(z))=f(z)[f(z+1)+f(z-1)]-C(z) f(z)-A(z) \equiv 0 .
$$

We notice that

$$
P_{1}(z, 0)=-A(z) \not \equiv 0 .
$$

Thus we deduce from Lemma 3.1 and (3.2) that

$$
m\left(r, \frac{1}{f}\right)=S(r, f)
$$

Therefore,

$$
N\left(r, \frac{1}{f}\right)=T(r, f)+S(r, f)
$$

and so $\lambda(f)=\sigma(f)$.

Now we prove $\lambda\left(\frac{1}{f}\right)=\sigma(f)$. By (3.1), we have

$$
f(z)[f(z+1)+f(z-1)]=C(z) f(z)+A(z) .
$$

Set $\sigma(f)=\sigma<\infty$. Then, by applying Lemma 3.2 to (3.3), we obtain

$$
m(r, f(z+1)+f(z-1))=O\left(r^{\sigma-1+\varepsilon}\right)+S(r, f),
$$

possibly outside of an exceptional set of finite logarithmic measure.

On the other hand, we conclude from the Valiron-Mohon'ko lemma and (3.1) that

$$
T(r, f(z+1)+f(z-1))=T\left(r, \frac{A(z)}{f(z)}+C(z)\right)=T(r, f)+S(r, f) .
$$

Hence, we deduce from Lemma 3.3, (3.4), (3.5), and the fact $\lambda=\lambda\left(\frac{1}{f}\right) \leq \sigma(f)=\sigma<\infty$ that

$$
\begin{aligned}
2 N(r, f) & \geq N(r, f(z+1)+f(z-1))+O\left(r^{\lambda-1+\varepsilon}\right)+O(\log r) \\
& =T(r, f)+O\left(r^{\sigma-1+\varepsilon}\right)+S(r, f),
\end{aligned}
$$

possibly outside of an exceptional set of finite logarithmic measure. Therefore, $\lambda\left(\frac{1}{f}\right)=\sigma(f)$.

(ii) Suppose that $f(z)$ is a finite order transcendental meromorphic solution of (3.1). If the conclusion does not hold, then there exists a finite value $b$ such that $\lambda(f-b)<\sigma(f-b)=$ $\sigma(f)<\infty$. This shows that there exists $\sigma \in \mathbb{N}$ such that $\sigma(f-b)=\sigma(f)=\sigma<\infty$. Thus, we can write $f(z)-b$ in the form

$$
f(z)-b=h(z) e^{d z^{\sigma}}
$$


where $d \neq 0$ is a constant, $h(z)$ is a meromorphic function satisfying

$$
\sigma(h)<\sigma(f)=\sigma
$$

We further conclude from (3.6) that

$$
f(z+1)=b+h(z+1) h_{+1} e^{d z^{\sigma}}, \quad f(z-1)=b+h(z-1) h_{-1} e^{d z^{\sigma}}
$$

where

$$
h_{+1}=\exp \left\{d \sum_{j=1}^{\sigma}\left(\begin{array}{c}
\sigma \\
j
\end{array}\right) z^{\sigma-j}\right\}, \quad h_{-1}=\exp \left\{d \sum_{j=1}^{\sigma}(-1)^{j}\left(\begin{array}{c}
\sigma \\
j
\end{array}\right) z^{\sigma-j}\right\} .
$$

Now substituting (3.6) and (3.8) into (3.1), we conclude that

$$
\begin{aligned}
& h(z) {\left[h(z+1) h_{+1}(z)+h(z-1) h_{-1}(z)\right] e^{2 d z^{\sigma}} } \\
& \quad+\left\{b\left[h(z+1) h_{+1}(z)+h(z-1) h_{-1}(z)\right]\right. \\
&\quad+(2 d-C(z)) h(z)\} e^{d z^{\sigma}}+2 d^{2}-d C(z)-A(z) \equiv 0 .
\end{aligned}
$$

We now apply Lemma 3.4 to (3.10) to obtain

$$
h(z)\left[h(z+1) h_{+1}(z)+h(z-1) h_{-1}(z)\right] \equiv 0 .
$$

This is impossible since $h(z), h(z+1), h(z-1), h_{+1}(z)$, and $h_{-1}(z)$ satisfy (3.6)-(3.9). Otherwise, if (3.11) holds, then we deduce from (3.1) and (3.8) that

$$
f(z)=\frac{A(z)}{2 b-C(z)} .
$$

This is a contradiction since $f(z)$ is transcendental and $A(z), C(z) \in \mathfrak{F}(f)$.

Hence, together with the result of (i), $f(z)$ has no Borel exceptional value.

(iii) Suppose that $f(z)$ is a finite order transcendental meromorphic solution of (3.1). Set

$$
g(z)=f(z)-z
$$

Then $g(z)$ is a finite order transcendental meromorphic function with

$$
\sigma(g)=\sigma(f)<\infty \quad \text { and } \quad \tau(f)=\lambda(g) .
$$

We substitute $f(z)=g(z)+z$ into (3.1) and obtain

$$
\begin{aligned}
P_{2}(z, g(z)):= & {[g(z)+z][g(z+1)+g(z-1)] } \\
& +(2 z-C(z)) g(z)+2 z^{2}-z C(z)-A(z) \\
\equiv & 0 .
\end{aligned}
$$


Since $P_{2}(z, 0)=2 z^{2}-z C(z)-A(z) \not \equiv 0$, we apply Lemma 3.1 to (3.12) to obtain

$$
N\left(r, \frac{1}{g}\right)=T(r, f)+S(r, f) .
$$

Therefore $\tau(f)=\lambda(g)=\sigma(f)$. The proof of Theorem 3.1 is completed.

\section{Competing interests}

The authors declare that they have no competing interests regarding the publication of this paper.

\section{Authors' contributions}

Both authors drafted the manuscript and approved the final manuscript.

\section{Author details}

'Department of Applied Mathematics, South China Agricultural University, Guangzhou, 510640, P.R. China. ${ }^{2}$ School of Mathematical Sciences, South China Normal University, Guangzhou, 510631, P.R. China.

\section{Acknowledgements}

Our research is supported by the Guangdong National Natural Science Foundation (No. 2014A030313422) and also partly supported by the National Natural Science Foundation of China (No. 11171119).

Received: 19 December 2014 Accepted: 25 August 2015 Published online: 04 September 2015

\section{References}

1. Hayman, WK: Meromorphic Functions. Clarendon, Oxford (1964)

2. Yang, L: Value Distribution Theory and New Research. Science Press, Beijing (1982) (in Chinese)

3. Halburd, RG, Korhonen, RJ: Finite-order meromorphic solutions and the discrete Painlevé equations. Proc. Lond. Math. Soc. 94(2), 443-474 (2007)

4. Grammaticos, B, Ramani, A, Papageorgiou, V: Do integrable mappings have the Painlevé property? Phys. Rev. Lett. 67(14), 1825-1828 (1991)

5. Halburd, RG, Korhonen, RJ: Difference analogue of the lemma on the logarithmic derivative with applications to difference equations. J. Math. Anal. Appl. 314(2), 477-487 (2006)

6. Chiang, YM, Feng, SJ: On the Nevanlinna characteristic of $f(z+\eta)$ and difference equations in the complex plane. Ramanujan J. 16(1), 105-129 (2008)

7. Ablowitz, MJ, Halburd, R, Herbst, B: On the extension of Painlevé property to difference equations. Nonlinearity 13(3), 889-905 (2000)

8. Bergweiler, W, Langley, JK: Zeros of differences of meromorphic functions. Math. Proc. Camb. Philos. Soc. 142(1), 133-147 (2007)

9. Chen, ZX, Shon, $\mathrm{KH}$ : Value distribution of meromorphic solutions of certain difference Painlevé equations. J. Math. Anal. Appl. 364(2), 556-566 (2010)

10. Halburd, RG, Korhonen, RJ: Nevanlinna theory for the difference operator. Ann. Acad. Sci. Fenn., Math. 31(2), $463-478$ (2006)

11. Halburd, RG, Korhonen, RJ: Existence of finite-order meromorphic solutions as a detector of integrability in difference equations. Physica D 218(2), 191-203 (2006)

12. Heittokangas, J, Korhonen, RJ, Laine, I, Rieppo, J, Tohge, K: Complex difference equations of Malmquist type. Comput. Methods Funct. Theory 1(1), 27-39 (2001)

13. Laine, I, Yang, CC: Clunie theorems for difference and q-difference polynomials. J. Lond. Math. Soc. 76(3), 556-566 (2007)

14. Yang, CC, Yi, HX: Uniqueness Theory of Meromorphic Functions. Kluwer Academic, Dordrecht (2003)

\section{Submit your manuscript to a SpringerOpen ${ }^{\circ}$ journal and benefit from:}

- Convenient online submission

Rigorous peer review

- Immediate publication on acceptance

- Open access: articles freely available online

- High visibility within the field

- Retaining the copyright to your article 